\title{
Clinical effects of FURL and PCNL with holmium laser for the treatment of kidney stones
}

\author{
ZHI-GANG LI, YAN ZHAO, TAO FAN, LIN HAO, CONG-HUI HAN and GUANG-HUI ZANG \\ Department of Urology, Xuzhou Central Hospital, Xuzhou, Jiangsu 221009, P.R. China
}

Received April 4, 2016; Accepted September 14, 2016

DOI: 10.3892/etm.2016.3835

\begin{abstract}
In the present study, the clinical effects of flexible ureteroscopy lithotripsy (FURL) and percutaneous nephrolithotomy (PCNL) for the treatment of kidney stones of $\leq 2 \mathrm{~cm}$ was studied. Seventy-two patients with kidney stones were randomly divided into the FURL group $(n=39)$ under ureteroscope lithotripsy with holmium laser and PCNL group $(n=33)$ under PCNL with holmium laser and compared their clinical effects. At 3 months after the operation, the stone removal rate of the FURL group was significantly higher than that of the PCNL group. The subgroup analysis revealed that the difference in the lower kidney calyx was more obvious $(\mathrm{P}<0.05)$ while the difference in the complex kidney stones was not statistically significant $(\mathrm{P}>0.05)$. The incidence of complications of the FURL group was significantly lower than that of the PCNL group $(\mathrm{P}<0.05)$. The operation time and recurrence rate of the FURL group were significantly less than that of the PCNL group $(\mathrm{P}<0.05)$. Differences regarding the creatinine and urea nitrogen levels before operation, and 3 and 7 days after the operation between the two groups were not statistically significant $(\mathrm{P}>0.05)$. Additionally, 3 and 7 days after operation, the cystatin $\mathrm{C}$ levels of the FURL group were significantly higher than those of the PCNL group, and the KIM-1 levels were significantly lower than the PCNL group $(\mathrm{P}<0.05)$. In conclusion, compared with PCNL with holmium laser, FURL with holmium laser was more safe and effective in treating kidney stones $\leq 2 \mathrm{~cm}$. Therefore, the method is worthy of wide application in clinic.
\end{abstract}

\section{Introduction}

Kidney stone is one of the three common diseases in urology, accounting for $80-90 \%$ of all urinary calculi (1).

Correspondence to: Dr Guang-Hui Zang, Department of Urology, Xuzhou Central Hospital, 199 Jiefang South Road, Xuzhou, Jiangsu 221009, P.R. China

E-mail: cbacw693708@163.com

Key words: flexible ureteroscopy lithotripsy, percutaneous nephrolithotomy, kidney stone, cystatin C, KIM-1
At present, the treatment of kidney stones has changed from open surgery to minimally invasive surgery, including extracorporeal shock wave lithotripsy (ESWL), percutaneous nephrolithotomy (PCNL) and flexible ureteroscope lithotripsy (FURL) (2,3). Although most kidney stones can be treated by ESWL, the curative effects are affected by many factors including the composition, size and location of the stones (4). In theory, PCNL may be used to treat the majority of kidney stones, especially stones $>2 \mathrm{~cm}$ in diameter (5). Although PCNL is very effective in treating kidney stones, it is also accompanied with great kidney damage and many complications (6). FURL is advantageous in its minimal invasiveness, safety, rapid recovery and high efficiency as it is a natural orifice performed under direct vision. It is extremely effective for stones that are $\leq 2 \mathrm{~cm}$ in diameter and complex kidney stones, and suitable for elderly patients, obese patients, patients with hemorrhagic disorders and patients that are not suitable for ESWL or PCNL $(7,8)$. In the clinic, many studies have been carried out to compare and analyze the curative effects of PCNL and FURL $(9,10)$.

In the present study, the clinical effects of FURL and PCNL with holmium laser for the treatment of kidney stones $\leq 2 \mathrm{~cm}$ were compared, and their impacts were analyzed, to provide a more scientific and accurate basis for clinical treatment.

\section{Patients and methods}

Patients. Seventy-two patients diagnosed with simple kidney stones by Kub, ultrasound or computed tomography (CT) at the Xuzhou Central Hospital (Jiangsu, China) from October, 2012 to October, 2014 were selected for the present study. The inclusion criteria for the study were: i) Age, $\geq 18$ and $<75$ years; ii) simple kidney stones; and iii) first treatment. The exclusion criteria were: i) Patients with complex kidney stones, combing ureteral stones, bladder stones, renal tuberculosis, renal tumor, renal dysfunction, acute and chronic nephritis, and nephrotic syndrome; ii) obese patients, patients with severe heart, liver, blood system diseases, and urinary system abnormalities; and iii) pregnant patients, with poor compliance or incomplete clinical data, or those who interrupted treatment.

Approval for the study was obtained from the Ethics Committee of the Xuzhou Central Hospital. Informed consent was obtained from the patients as well as their relatives. Subsequently, the patients were randomly divided into the FURL group $(n=39)$ under ureteroscope lithotripsy with 
holmium laser and the PCNL group (n=33) under PCNL with holmium laser. In the FURL group, there were 22 men and 17 women (age range, 36-65 years), with an average age of $49.7 \pm 10.2$ years. The patients had a kidney stone of $1.2-1.9 \mathrm{~cm}$ in diameter and an average of $1.6 \pm 0.4 \mathrm{~cm}$, with 15 stones on the left side, 17 on the right side and 7 on both sides. The stone

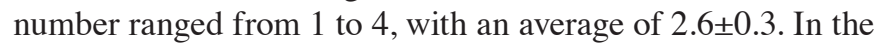
PCNL group, there were 19 men and 14 women, (age range, 38-69 years), and an average age of $52.3 \pm 11.4$ years. The kidney stone was $1.1-1.9 \mathrm{~cm}$ in diameter, with an average size of $1.5 \pm 0.5 \mathrm{~cm}$. Thirteen stones were on the left side, 15 on the right side and 5 on both sides. The stone number ranged from 1 to 4 , with an average of 2.3 \pm 0.4 . Differences on the basic data of the two groups were not statistically significant $(\mathrm{P}>0.05)$.

Major surgical instruments. The instruments used in the present study were: URF-P5 flexible ureteroscope (Olympus, Tokyo, Japan), flexible laser (200 pm, holmium laser fiber) (Lumenis, Beijing, China), 0.035 foot nickel titanium super smooth guide wire $(0.888 \mathrm{~mm}$ x $150 \mathrm{~cm})$ (C.R. Bard, Inc., Murray Hill, NJ, USA), 8.5/9.8 rigid ureteroscope and 20.8 nephroscope (both from Richard Wolf GmbH, Knittlingen, Germany), and a renal puncture suit (Urovision GmbH, Achenmühle, Germany).

Surgical procedures of ureteroscope lithotripsy group. After general anesthesia, while keeping patients in the lithotomy position the F8/9.8 wolf flexible ureteroscope was inserted through the urine tract under direct vision. It was followed by the interureteric ridge and the ureterostoma of the affected side was located to insert the rigid ureteroscope into the ureter of the affected side. Subsequently, the ureter was observed and expanded. Retrogradely, the head or renal pelvis of ureter was indwelled with 0.035 nickel titanium super smooth guide wire, and then the rigid ureteroscope was removed. The flexible ureteroscope sheath was placed into ureter along the super smooth guide wire, and the super smooth guide wire was removed. A channel from the external urethral orifice was established to renal pelvis through the flexible ureteroscope sheath. The flexible ureteroscope was inserted along with the flexible ureteroscope sheath under direct vision. Subsequently, the renal pelvis and renal calyx was comprehensively observed and the stone was located to observe size and number. The holmium laser fiber was connected with the stone crusher and power was set at 0.8-1.0 J/15-20 Hz, to embed the holmium laser fiber through the working channel of the flexible ureteroscope and the fiber exposed approximately $0.5-1 \mathrm{~cm}$ over the front end of the working channel in order that head of the fiber was visible on the display. The lens of the flexible ureteroscope and angle of the fiber was adjusted to start breaking the stones. During the operation, smooth flush fluid was maintained to avoid excessive perfusion pressure. After breaking stones, the renal pelvis and renal calyx was examined for any abnormality, and then F5 double J tube was retained. After the operation, the patients were treated with conventional antibiotics for $48 \mathrm{~h}$ and re-examined KUB 3 days after the operation to observe stone breakage and location of the double $\mathrm{J}$ tube. The double $\mathrm{J}$ tube was removed after 2-4 weeks.

Surgical procedures of the PCNL group. After general anesthesia, while keeping patients in the prone position, the abdomen of the renal region was raised by $10-15 \mathrm{~cm}$ to make the waist and back of the affected side at a low arch at an angle of $30^{\circ}$ with the operating table. A puncture region was made to the funnel-shaped fluid collection bag and ultrasound was performed to examine the state of kidney in the affected side and objective renal calyx was chosen. If there was any urine flow after the pin core was removed, it proved that puncture was successful. The safe guiding wire was implanted and fixed well. It was expanded to F16 along the safe guiding wire by fascia dilator, and the peel-away sheath was retained to establish a percutaneous renal passage. The rigid ureteroscope was inserted into the renal pelvic under the guidance of the guiding wire, and observed to locate the stones. Then, the holmium laser was used to break the stones, and the operations were conducted as mentioned above.

Observation indexes and detection methods. The differences of the stone removal rate, complication incidence, operation time, 1-year follow-up recurrence rate, creatinine level, urea nitrogen level, cystatin C level and KIM-1 level of the two groups was compared. The stone removal rate referred to no retained stones found or the fragments of retained stone of a size of $<4 \mathrm{~mm}$ and free from any clinical symptoms under KUB, ultrasound or CT examination within 3 months after the operation. Complications related to ureteroscope lithotripsy included injury, avulsion and perforation of renal pelvis and ureter mucosa avulsion, gross hematuria, renal colic, infection, formation of lithanguria, ureteral stent syndrome, ureteral stricture, and formation of ureteral stent surface stone. Complications related to PCNL included intraoperative and postoperative bleeding, urine leakage, perinephric abscess, urogenous septicopyemia and formation of renal cortex scar.

To detect renal function damage, $5 \mathrm{ml}$ fasting venous blood and $6 \mathrm{ml}$ urina sanguinis were collected. The blood was centrifuged at 2,500 x g for $5 \mathrm{~min}$ to collect the supernatant and preserved at $-80^{\circ} \mathrm{C}$. The conventional biochemical detector was used to detect the creatinine and urea nitrogen. ELISA was used to detect serum cystatin C and urine KIM-1 (kit by Hangzhou Dawen Biotech Co., Ltd., Hangzhou, China).

Statistical analysis. SPSS 19.0 software (IBM SPSS, Armonk, NY, USA) was used for the statistical analysis. Measurement data were presented as mean $\pm \mathrm{SD}$, and the t-test was used for comparison of groups. The data were presented as percentage $(\%)$, and the $\chi^{2}$ test was applied for comparison of groups. $\mathrm{P}<0.05$ was considered to indicate a statistically significant difference.

\section{Results}

Comparison of the stone removal rate. Differences on the stone removal rate of the two groups in 1 week and 1 month after the operation were not statistically significant $(\mathrm{P}>0.05)$. Three months after the operation, the stone removal rate of the FURL group was significantly higher than that of the PCNL group $(\mathrm{P}<0.05)$. According to the subgroup analysis, the difference in the lower kidney calyx was more obvious $(\mathrm{P}<0.05)$ while the difference in the complex kidney stones was not statistically significant $(\mathrm{P}>0.05)$ (Table I). 
Table I. Comparison of stone removal rate, case (\%).

\begin{tabular}{lcccccc}
\hline Groups & Cases & $\begin{array}{c}\text { 1 week after } \\
\text { operation }\end{array}$ & $\begin{array}{c}1 \text { month after } \\
\text { operation }\end{array}$ & $\begin{array}{c}3 \text { months after } \\
\text { operation }\end{array}$ & $\begin{array}{c}\text { Lower } \\
\text { calyx }\end{array}$ & $\begin{array}{c}\text { Complex kidney } \\
\text { stone }\end{array}$ \\
\hline FURL & 39 & $27(69.2)$ & $30(76.9)$ & $33(84.6)$ & $24 / 30(80.0)$ & $4 / 8(50.0)$ \\
PCNL & 33 & $19(57.6)$ & $20(60.6)$ & $21(63.6)$ & $14 / 26(53.8)$ & 4.368 \\
$\chi^{2}$ & & 1.052 & 2.243 & 4.196 & 0.037 & 0.246 \\
P-value & & 0.305 & 0.134 & 0.041 & 0.620 \\
\hline
\end{tabular}

FURL, flexible ureteroscope lithotripsy; PCNL, percutaneous nephrolithotomy.

Table II. Comparison of operation time, recurrence rate and complications.

\begin{tabular}{lcccc}
\hline Groups & Cases & Operation time $(\mathrm{min})$ & Recurrence rate, case $(\%)$ & Complication, case $(\%)$ \\
\hline FURL & 39 & $46.7 \pm 12.3$ & $3(7.7)$ & $6(15.4)$ \\
PCNL & 33 & $62.3 \pm 15.4$ & $4(12.1)$ & $12(6.4)$ \\
T $\left(\chi^{2}\right)$ & & 4.927 & 0.054 & 4.196 \\
P-value & & 0.035 & 0.816 & 0.041 \\
\hline
\end{tabular}

FURL, flexible ureteroscope lithotripsy; PCNL, percutaneous nephrolithotomy.

Comparison of operation time, recurrence rate and complications. The operation time and recurrence rate of the FURL group were significantly less than those of the PCNL group $(\mathrm{P}<0.05)$. In the FURL group, there were 3 cases of ureteral mucosa injury, 2 cases of gross hematuria and 1 case of ureteral stricture, and the incidence of complication was $15.4 \%$. In the PCNL group, there were 5 cases of bleeding and hematoma, 2 cases of urine leakage and 4 cases of renal abscess and infection, 1 case of renal cortex scar, and the incidence of complication was $36.4 \%$. The incidence of complications of the FURL group was significantly lower than that of the PCNL group $(\mathrm{P}<0.05)$, as shown in Table II.

Comparison of renal damage indexes. Differences on the creatinine and urea nitrogen levels before the operation, and 3 and 7 days after the operation between the two groups were not statistically significant $(\mathrm{P}>0.05)$. After 3 and 7 days of operation, the cystatin C levels of the FURL group were significantly higher than those of the PCNL group, and the KIM-1 levels were significantly lower than the PCNL group $(\mathrm{P}<0.05)$, as shown in Table III.

\section{Discussion}

FURL is a new minimally invasive technique of urology surgery. Along with the development of new ureteroscopes and progress of the manufacturing technology of ureteroscope-related accessory equipment, ureteroscopes are increasingly used in the diagnosis and treatment of upper urinary tract diseases, especially kidney stones (11). Holmium laser optic, basket extractor, nipper, and ureteroscope delivery sheath are tools for stone crush and removal. According to the study of Lomanto et al, holmium laser was an impulse mode solid laser system with a 2,140 nm wavelength that could effectively crush all urinary calculus and the stone fragments were smaller than other lithotripters for easy removal from the body, thus avoiding use of the basket extractor and other stone removal equipment (12).

Different studies have been carried out to explore the clinical effects of FURL on the treatment of kidney stones, but the results were contradictory. The present study innovatively combined holmium laser with the two surgical methods, which not only improved the success rate of surgery (12), but also avoided the system bias error resulting from the combination of the two surgical methods with other lithotripsies. By comparing the stone removal rate at different time-points and in different subgroups, the present study further explained the different effects of the two surgical methods. The results of the present study have shown that the stone removal rates of the two surgical methods were significantly different 3 months after surgery, indicating that the time of stone removal may affect the experiment and improving the stone removal conditions may increase the stone removal rate. The lithotrity effects of FURL were more obvious in lower calyx, consistent with the study results of Carrouget et al (13). FURL followed the direction of urinary tract and directly contacted pelvis and lower calyx. It was also effective for the stones of larger angle. It was reported (14) that FURL was effective for complex kidney stones. Since the sample size of our study was limited, no different conclusion was obtained. The average surgical time of FURL was significantly shortened, consistent with the study results of Unsal et al (15). After 1-year follow-up, it was found that the recurrence rate of the FURL group was significantly lower than that of the PCNL group, another innovation point of this study. Through detailed statistics of the incidence of complications, it was found that the incidence of complications of the FURL group was significantly lower than that of the FURL group, which was not analyzed in previous studies. 
Another innovative point of the present study was monitoring of renal damage. In the current study, we monitored, not only the traditional creatinine and urea nitrogen, but also the more sensitive and specific cystatin C and KIM-1. The results of the present study have shown that differences on the creatinine and urea nitrogen levels before operation, and 3 and 7 days after the operation between the two groups were not statistically significant. By contrast, 3 and 7 days after the operation, the cystatin C level of the FURL group was significantly higher than that of the PCNL group, and the KIM-1 level was significantly lower than that of the PCNL group, with differences being statistically significant.

KIM-1 is type I transmembrane glycoprotein and a member of the immunoglobulin ( $\mathrm{Ig}$ ) gene superfamily. Its expression had extremely high tissue specificity. It is expressed weakly in liver, kidney and spleen of healthy individuals but strongly expressed in kidney tissues with ischemic injury. When the top of epithelial cells of the proximal renal tubule was damaged, KIM-1 showed a high expression, which persisted before the epithelial cells of the damaged renal tubular recovered. Previous findings have shown that KIM-1 was expressed in urine and renal tubular epithelial cells in the early stage of renal function damage, and its expression was positively correlated with the severity of renal tissue pathology (16). Therefore, KIM-1 may be used as a sensitive index for the early diagnosis of renal tubular function. In addition, the specificity of urine KIM-1 was high and free from the impacts of physical and chemical properties of urine. Cystatin $\mathrm{C}$ is a non-glycosylated low protein with a low molecular weight, and was expressed in all the nucleated cells and produced speed stability. Cystatin C was not affected by age, gender, infection, tumor, diet, weight and liver function changes, most drugs or other factors. Kidney is the only organ that clears away cystatin $\mathrm{C}$ in circulation. It is metabolized by kidney, reabsorbed in proximal tubule cells and completely degraded in epithelial cells. It cannot return to the blood circulation nor be secreted by kidney tubules; consequently, cystatin $\mathrm{C}$ may be used as an endogenous marker of early GFR. When glomerulus was slightly damaged, the concentration of cystatin $\mathrm{C}$ increased after $3 \mathrm{~h}$, and its elevation level was positively correlated with the degree of glomerular injury. Cystatin $\mathrm{C}$ has become a recognized index for the early evaluation of renal function injury $(17,18)$.

In conclusion, compared with PCNL with holmium laser, FURL with holmium laser was more safe and effective in treating kidney stones $\leq 2 \mathrm{~cm}$, and is therefore worthy of wide application in clinic.

\section{References}

1. Moe OW: Kidney stones: pathophysiology and medical management. Lancet 367: 333-344, 2006.

2. Geavlete P, Multescu R and Geavlete B: Influence of pyelocaliceal anatomy on the success of flexible ureteroscopic approach. J Endourol 22: 2235-2239, 2008.

3. Srisubat A, Potisat S, Lojanapiwat B, Setthawong V, Schmidt S and Miernik A: [Extracorporeal shock wave lithotripsy (ESWL) versus percutaneous nephrolithotomy (PCNL) or retrograde intrarenal surgery (RIRS) for kidney stones]. Urologe A 54: 1283-1286, 2015.

4. Proietti S and Traxer O: The era of shock wave lithotripsy is over: Yes. J Urol 195: 17-18, 2016.

5. Datta SN, Solanki R and Desai J: Prospective outcomes of ultra mini percutaneous nephrolithotomy: a consecutive cohort study. J Urol 195: 741-746, 2016. 
6. Kyriazis I, Panagopoulos V, Kallidonis P, Özsoy M, Vasilas M and Liatsikos E: Complications in percutaneous nephrolithotomy. World J Urol 33: 1069-1077, 2015.

7. Hassan M, El-Nahas AR, Sheir KZ, El-Tabey NA, El-Assmy AM, Elshal AM and Shokeir AA: Percutaneous nephrolithotomy vs. extracorporeal shockwave lithotripsy for treating a 20-30 mm single renal pelvic stone. Arab J Urol 13: 212-216, 2015

8. Bagcioglu M, Demir A, Sulhan H, Karadag MA, Uslu M and Tekdogan UY: Comparison of flexible ureteroscopy and micropercutaneous nephrolithotomy in terms of cost-effectiveness: analysis of 111 procedures. Urolithiasis: Oct 16, 2015 (Epub ahead of print)

9. Marroig B, Frota R, Fortes MA, Sampaio FJ and Favorito LA: Influence of the renal lower pole anatomy and mid-renal-zone classification in successful approach to the calices during flexible ureteroscopy. Surg Radiol Anat 38: 293-297, 2016.

10. Wilhelm K, Frankenschmidt A and Miernik A: Analgesia-free flexible ureteroscopic treatment and laser lithotripsy for removal of a large urinary stone: a case report. J Med Case Rep 9: 225, 2015.

11. Tauber V, Wohlmuth M, Hochmuth A, Schimetta W and Krause FS: Efficacy management of urolithiasis: flexible ureteroscopy versus extracorporeal shockwave lithotripsy. Urol Int 95: 324-328, 2015.

12. Lomanto D, Fiocca F, Nardovino M, Grasso E, Lezoche E, Zarba Meli E, Paganini A and Speranza V: ESWL experience in the therapy of difficult bile duct stones. Dig Dis Sci 41: 2397-2403, 1996.
13. Carrouget J, Ammi M, Nedelcu C, Cassereau J, Lebdai S, Azzouzi AR and Bigot P: Posterior spinal cord syndrome after flexible ureteroscopy for kidney stones. Urol Int 96: 116-118, 2016.

14. Altay B, Erkurt B, Kiremit MC and Güzelburç V: A rare complication of ureteral double-J stenting after flexible ureteroscopy: renal parenchymal perforation. Turk J Urol 41: 96-98, 2015.

15. Unsal A, Resorlu B, Kara C and Bayindir M: The role of percutaneous nephrolithotomy in the management of mediumsized $(1-2 \mathrm{~cm})$ lower-pole renal calculi. Acta Chir Belg 111: 308-311, 2011.

16. Hyams ES, Munver R, Bird VG, Uberoi J and Shah O: Flexible ureterorenoscopy and holmium laser lithotripsy for the management of renal stone burdens that measure 2 to $3 \mathrm{~cm}$ : a multi-institutional experience. J Endourol 24: 1583-1588, 2010.

17. Akdeniz D, Celik HT, Kazanci F, Yilmaz H, Yalcin S, Bilgic MA, Ruzgaresen N, Akcay A and Eryonucu B: Is kidney injury molecule 1 a valuable tool for the early diagnosis of contrast-induced nephropathy? J Investig Med 63: 930-934, 2015.

18. Deyà-Martínez À, Fortuny $\mathrm{C}$, Soler-Palacín $\mathrm{P}$, Neth $\mathrm{O}$, Sánchez E, Martín-Nalda A, Falcón-Neyra L, Vila A, Valls A and Noguera-Julian A: Cystatin C: a marker for inflammation and renal function among HIV-infected children and adolescents. Pediatr Infect Dis J 35: 196-200, 2016. 